\title{
Ex vivo Sensitivity Profile of Plasmodium falciparum Clinical Isolates to a Panel of Antimalarial Drugs in Ghana 13 Years After National Policy Change
}

This article was published in the following Dove Press journal: Infection and Drug Resistance

\author{
Michael Fokuo Ofori $\mathbb{D}^{1, *}$ \\ Emma E Kploanyi iD \\ Benedicta A Mensah ${ }^{2}$ \\ Emmanuel K Dickson' \\ Eric Kyei-Baafour ${ }^{\prime}$ \\ Sampson Gyabaa ${ }^{3}$ \\ Mary Tetteh ${ }^{4}$ \\ Kwadwo A Koram (iD ${ }^{2}$ \\ Benjamin K Abuaku ${ }^{2, *}$ \\ Anita Ghansah ${ }^{1,5, *}$ \\ 'Immunology Department, Noguchi \\ Memorial Institute for Medical Research, \\ University of Ghana, Legon, Accra, Ghana; \\ ${ }^{2}$ Epidemiology Department, Noguchi \\ Memorial Institute for Medical Research, \\ University of Ghana, Legon, Accra, Ghana; \\ ${ }^{3}$ Ewim Polyclinic, Ghana Health Service, \\ Cape Coast, Ghana; ${ }^{4}$ Begoro District \\ Hospital, Ghana Health Service, Begoro, \\ Ghana; ${ }^{5}$ Parasitology Department, \\ Noguchi Memorial Institute for Medical \\ Research, University of Ghana, Legon, \\ Accra, Ghana
}

*These authors contributed equally to this work
Correspondence: Michael Fokuo Ofori Immunology Department, Noguchi Memorial Institute for Medical Research, University of Ghana, Post Office Box

LG58I, Legon, Ghana

Tel +233244715975

Fax +233302502182

Email mofori@noguchi.ug.edu.gh
Purpose: Malaria continues to be a major health issue globally with almost $85 \%$ of the global burden and deaths borne by sub-Saharan Africa and India. Although the current artemisinin derived combination therapies in Ghana are still efficacious against the Plasmodium falciparum ( $P f$ ) parasite, compounding evidence of artemisinin and amodiaquine resistance establish the need for a full, up-to-date understanding and monitoring of antimalarial resistance to provide evidence for planning control strategies.

Materials and Methods: The study was cross-sectional and was conducted during the peak malaria transmission seasons of 2015, 2016, and 2017 in two ecological zones of Ghana. Study participants included children aged 6 months to 14 years. Using ex vivo 4,6-diamidino-2-phenylindole (DAPI) drug sensitivity assay, $330 \mathrm{Pf}$ isolates were used to investigate susceptibility to five antimalarial drugs: chloroquine (CQ), amodiaquine (AMD) dihydroartemisinin (DHA), artesunate (ART) and mefloquine (MFQ).

Results: The pooled geometric mean $\mathrm{IC}_{50} \mathrm{~S}\left(\mathrm{GMIC}_{50}\right)$ of the five drugs against the parasites from Cape Coast and Begoro were 15.5, 42.4, 18.9, 4.6 and 27.3nM for CQ, AMD, DHA, ART, and MFQ, respectively. The $\mathrm{GMIC}_{50}$ values for CQ $(\mathrm{p}<0.001)$, ART $(\mathrm{p}<0.011)$ and DHA $(\mathrm{p}<0.018)$ were significantly higher for Cape Coast isolates as compared to Begoro isolates. However, $\mathrm{GMIC}_{50}$ estimates for MFQ $(\mathrm{p}<0.022)$ were significantly higher for Begoro isolates. Positive correlations were found between each pair of drugs with the weakest found between MFQ and DHA $(r=0.34 ; \mathrm{p}<0.001)$, and the strongest between ART and DHA $(r=0.66 ; \mathrm{p}<0.001)$.

Conclusion: The parasites showed reduced sensitivities to three (AMD, DHA and MFQ) out of the five drugs assessed. The study also demonstrated the continual return of chloroquine-sensitive parasites after 13 years of its withdrawal as the first-line drug for the treatment of uncomplicated malaria in Ghana. The ex vivo DAPI assay is a reliable method for assessing antimalarial drug sensitivities of $P f$ field isolates under field settings.

Keywords: malaria, Plasmodium falciparum, anti-malarial drugs, field isolates, DAPI, uncomplicated malaria

\section{Introduction}

Malaria continues to be a major health issue globally and efforts are being invested to either control or eliminate it in most endemic regions of the world. The World Health Organization (WHO) has reported that globally, an estimated 93\% of all malaria cases, 99.7\% of which are Plasmodium falciparum (Pf) infections, and 94\% of all malaria deaths occur in the African region. ${ }^{1}$ Almost $85 \%$ of the global malaria burden and 
deaths were borne by some countries in sub-Saharan Africa and India with Nigeria alone accounting for about $25 \%$ of the malaria cases and $24 \%$ of all global malaria deaths. Apart from Nigeria, Ghana reported the highest absolute increases in cases of malaria in 2018 compared with 2017 among the 10 highest burden countries in Africa.

The exposure of malaria parasites to sub-optimal drug concentrations increases the possibility of malaria drug resistance $^{2}$ and it has been suggested to occur simultaneously with the introduction of the drugs. ${ }^{3}$ Resistance has emerged to all antimalarial drugs ever prescribed for the treatment of malaria. ${ }^{4}$ Chloroquine (CQ) and Sulfadoxine/Pyrimethamine (SP) resistance were the first to be documented which mainly affected Southeast Asia, Africa, South America, and some Pacific regions.

Artemisinin (ARS) and its derivatives are currently the most potent and rapidly acting antimalarials, recommended by WHO as component of first-line combination therapy for Falciparum malaria in all endemic countries. ${ }^{5-7}$ Unfortunately, recent alarming findings indicate the emergence of ARSresistant parasites in Southeast Asia, the same place CQ and SP resistance originated from and spread in Africa. ${ }^{8,9}$ Even more alarming are the compounding evidence of reduced susceptibility of $P f$ to Artesunate (ART) in Cambodia and the Thai-Myanmar border. ${ }^{5,10}$ Increased resistance to Amodiaquine (AMD) has also been documented in Afghanistan ${ }^{11}$; first recorded in the African region in Tanzania as early as 2004, then in Angola ${ }^{12}$ and more recently in Southwestern Nigeria. ${ }^{13}$ Also, findings suggest reduced $P f$ susceptibility to the Artesunate-Mefloquine (ART-MFQ) combination therapy in the Cambodia - Thailand border ${ }^{14,15}$ and Benin. ${ }^{16}$ Besides, resistance to AMD has not been contained and has now spread beyond Southeast Asia. ${ }^{17}$ These findings raise great concern particularly for Africa, where the spread of resistant $P f$ strains brings devastating outcomes. ${ }^{18}$ Therefore, there is a need for a full, up-to-date understanding and monitoring of antimalarial resistance to provide evidence for planning control strategies. Controlling the spread of antimalarial drug resistance, especially resistance of $P f$ to artemisininbased combination therapies, is a high priority because drug resistance has often threatened malaria elimination efforts. ${ }^{6}$

Current methods recommended by WHO for monitoring antimalarial drug resistance are: in vivo therapeutic efficacy testing, in vitro studies to measure intrinsic sensitivity of antimalarial drugs and molecular markers to identify genetic mutations associated with antimalarial drug resistance. These methods complement each other to provide timely information for national malaria control programme-based treatment policy. ${ }^{19}$ In Ghana, subsequent to $C Q$ resistance, the Artesunate-Amodiaquine (ART-AMD) combination therapy was adopted for uncomplicated malaria in 2004. This treatment regimen has proven effective for over 10 years and remains efficacious. ${ }^{20,21}$ However, the compounding evidence of ARS and AMD resistance in other countries within the Sub-region including Nigeria ${ }^{14}$ and Benin ${ }^{17}$ warrants the continuous monitoring and evaluation of malaria drug resistance (MDR) especially when the treatment program has been running for over a decade as MDR has often threatened malaria elimination efforts. On the other hand, $P f$ 's susceptibility to CQ after removal of drug pressure also has to be monitored. ${ }^{22}$ It is also important to monitor drug resistance in areas of varying transmission intensity across the country to indicate hotspots of drug resistance for effective control. ${ }^{23}$

This study applied the in vitro DAPI assay to study isolates from two study sites (different transmission intensity and patterns) using a panel of drugs currently administered in Ghana. In addition, these isolates were tested with previously used CQ to investigate if chloroquine - sensitive field isolates have return after its removal as the first line of drug for the treatment of uncomplicated malaria in Ghana.

\section{Materials and Methods Ethical Statement}

The study complied with the declaration of Helsinki and was approved by the Noguchi Memorial Institute for Medical Research Institutional Review Board (NMIMR IRB No. 056/12-13). Study aims and objectives, benefits and possible risks were explained to all the parents/guardians of the study participants. Written informed consent was obtained from parents/guardians of each participant prior to enrolment. Additionally, written informed assent was obtained from participants aged 12 years and above.

\section{Study Area and Population}

The study was part of a therapeutic efficacy hospital-based study that targeted children 6 months to 14 years and was conducted in two sites in Ghana with different malaria transmission patterns - the Begoro District Hospital in the Eastern region and Ewim polyclinic in the Central region. The Begoro District Hospital is situated in the Fanteakwa District within the Forest ecological zone of Ghana. The District has an annual rainfall of $1500-2000 \mathrm{~mm}$ with malaria transmission being intense and perennial. ${ }^{24-26}$ The Ewim Polyclinic is situated in 
the Cape Coast Metropolis within the coastal Savannah zone of Ghana where transmission is low to moderate and also perennial with an annual rainfall of $750-1000 \mathrm{~mm}^{27}$ (Figure 1). The malaria transmission pattern is similar to what pertains in other parts of Ghana with two seasonal peaks - major and minor. The major season occurs in April-July with the minor occurring in September-November. ${ }^{28}$ The study involved children aged 6 months -14 years presenting with a history of fever, monoinfection with Plasmodium falciparum parasite density of $1000-250,000 / \mu \mathrm{L}$ blood; and absence of severe malaria. All the children diagnosed with mix infection by microscopy were excluded from the study. After meeting the inclusion criteria and informed consent obtained from parents with assent from children 12 years and above, $4 \mathrm{~mL}$ of blood was aseptically obtained from each study participant of which $400 \mu \mathrm{L}$ was used for the in vitro drug sensitivity assay.

\section{DAPI Drug Assay}

\section{Drugs}

The antimalarial drugs used in this study included chloroquine (CQ), amodiaquine (AMD), artesunate (ART), mefloquine (MFQ) and dihydroartemisinin (DHA). These drugs were

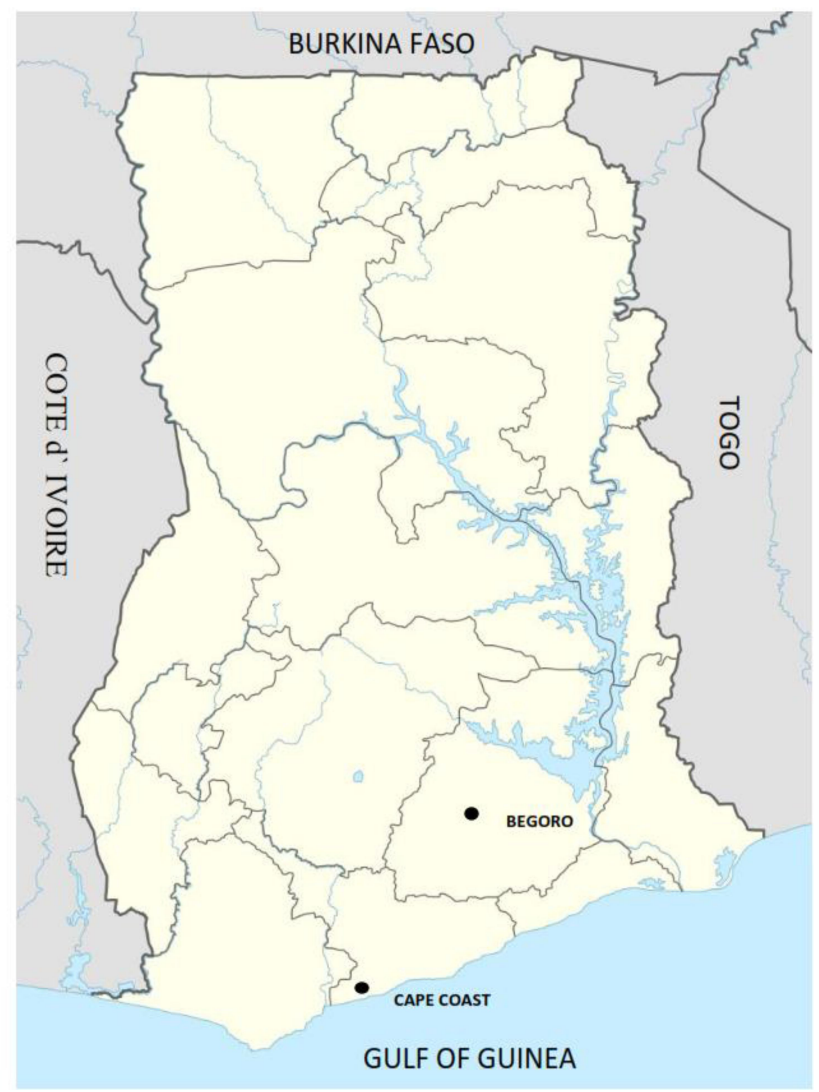

Figure I The map of Ghana showing the two study sites (black dot) within different ecological zones: Begoro in the forest area and Cape Coast in the coastal savanna area. obtained from Wirth Group at the Harvard T.N. Chan School of Public Health (gift to Anita Ghansah). Preparation: The stock concentration of chloroquine was first prepared in sterile distilled water and then DMSO whereas the rest of the drugs were prepared in DMSO. Aliquots of $20 \mu \mathrm{L}$ of drug were added in duplicates with two-fold dilution to their respective wells in three 96-welled black with clear flat bottom tissue culture treated microtiter plates (Corning, USA) according to working concentration ranging from $750 \mathrm{nM}$ to 2.93 (CQ), 250 to $0.98 \mathrm{nM}$ (AMD), 150 to $0.59 \mathrm{nM}$ (ART), 75 to $0.29 \mathrm{nM}$ (DHA), and 500 to $1.95 \mathrm{nM}$ (MFQ).

\section{In vitro Drug Sensitivity Assay}

Parasitized blood samples collected from patients enrolled were washed twice with $10 \mathrm{~mL}$ incomplete medium (without normal human serum) that ensured the complete removal of white blood cells including leucocytes. In brief, the heparinized blood was initially centrifuged at 2000rpm for 10 minutes and the plasma and the buffy coats were removed. The packed RBCs were then re-suspended in incomplete medium and again span at $2000 \mathrm{rpm}$ for 10 minutes and the supernatants together with some buffy coats and topmost part of the parasitized RBCs discarded. The step was repeated until not white layer observed after washing as described. The erythrocytes were re-suspended at $2 \%$ hematocrit in complete medium containing supplemented RPMI 1640, $2 \%$ normal human serum, $10 \%$ Albumax and $10 \mathrm{mg} / \mathrm{mL}$ gentamycin. Blood samples with parasitemia higher than $1 \%$ were adjusted to $1 \%$ with leucocyte-free uninfected $\mathrm{O}^{+}$ erythrocytes. For each sample, aliquots of $180 \mu \mathrm{L}$ of parasite culture were added to the pre-dosed drugs in duplicates in a set of three plates with a negative control and incubated at $37^{\circ} \mathrm{C}$ for 72 hours in an airtight chamber containing a gas mixture of $5.5 \% \mathrm{CO}_{2}, 2 \% \mathrm{O}_{2}$ and $92.5 \% \mathrm{~N}_{2}$. The remaining parasite cultures were maintained under the same conditions in T25 culture flasks for daily monitoring which included change of media and preparation of smears from the culture for observing $P f$ growth using a light microscope. After 72 hours, the processed samples in the plates were harvested by preparing smears from a control well in each plate after which the plates were wrapped in aluminum foil and stored in a freezer at $-20^{\circ} \mathrm{C}$ until ready to be read.

\section{DAPI Plasmodium falciparum Growth Assay}

A non-radioactive fluorescence-based procedure was conducted using a protocol adapted by Ndiaye et $\mathrm{al}^{29}$ for 
frozen plate assay. The frozen plates were allowed to thaw at room temperature and spun for 30 minutes at $4000 \mathrm{rpm}$. The contents of these plates were discarded and padded on tissue to absorb all well content except the $P f$ DNA which settled as pellets in the wells. Aliquots of $100 \mu \mathrm{L}$ of fluorochrome mixture containing $20 \mathrm{mM}$ Tris- $\mathrm{HCl}(\mathrm{pH} 7.5)$ (MP Biomedicals, LLC), 5 mM EDTA (Sigma-Aldrich), 0.004\% Saponin (Sigma Life Science), 0.01\% Triton X-100 (Sigma Life Science), and a 1:75,000 final dilution of $5 \mathrm{mg} / \mathrm{mL}$ DAPI (Sigma Life Science) were added to each well containing $P f$ DNA using a multi-channel pipette. This was carried out in the dark since the stain is light sensitive. The DAPI-Buffer-Pf DNA mixture was resuspended in each well using a multi-channel pipette after which the plates were wrapped in aluminium foil to protect them from light and left to incubate at room temperature in the dark for 30 minutes. The incubation was followed with spinning for another 30 minutes at 4000rpm and well contents were discarded. Aliquots of $200 \mu \mathrm{L}$ of $1 \times$ PBS were added per well using a multi-channel pipette with re-suspension. These plates were kept covered with aluminium foil until reading. The fluorescence from the stained parasite DNA in each plate well was measured using Tecan infinite M200PRO (Ex/Em: 358/461nm, bound to DNA).

\section{Statistical Analysis}

The GMIC $_{50}$ (the drug concentration inhibiting $50 \%$ of parasite activity) for each drug was estimated from the fluorescence data generated using the online tool, ICE estimator $1.2 .{ }^{30} \mathrm{GMIC}_{50}$ values with their $95 \%$ confidence intervals (CI) were calculated by using an Emax model available at http://www.antimalarial-icestimator.net as RE $=100-[(100 * \mathrm{C} \gamma) /(\mathrm{C} \gamma+\mathrm{IC} \gamma)]$, where $\gamma$ is a sigmoidicity factor which expresses the steepness of the curve, RE is the relative effect of the drug (in percent, Y-axis), and $\mathrm{C}$ is the drug concentration (X-axis). The estimated drug GMIC $_{50}$ were compared between two study sites using Mann-Whitney test All statistical analysis and figures were done using the software $\mathrm{R}$ and GraphPad Prism, respectively. Statistical tests were assumed significant at $\mathrm{P}<0.05$.

\section{Results}

A DAPI-based in vitro assay was carried out to monitor the drug sensitivity of $330 \mathrm{Pf}$ clinical isolates. The assay successfully tested an average of $58.6 \%$ (range: $53 \%$ - 65\%) of clinical isolates over the 3 -year period (Table 1). Generally, higher success rates were recorded among isolates collected from Cape Coast against all the drugs except that of artesunate for which Begoro isolates showed a higher success rate. As internal control, all samples which did not show appreciable parasite growth in culture (unsuccessful growth) were eliminated from subsequent analysis.

Comparison between $\mathrm{GMIC}_{50}$ values for the five drugs estimated for the two different transmission zones: Begoro and Cape Coast. The $\mathrm{GMIC}_{50}$ values for CQ were $11.90 \mathrm{nM}$ and $17.67 \mathrm{nM}$ for Begoro and Cape Coast, respectively $(\mathrm{p}<0.001)$, ART $3.77 \mathrm{nM}$ versus $5.21 \mathrm{nM}(\mathrm{p}<0.011)$ and DHA $14.50 \mathrm{nM}$ against $21.15 \mathrm{nM}$, respectively $(\mathrm{p}<0.018)$, for Begoro and Cape Coast isolates isolates, respectively (Figure 2 and Supplementary Table S1). However, GMIC 50 estimates for MFQ $(\mathrm{p}<0.022)$ were significantly higher against the Begoro isolates as compared to the Cape Coast isolates $(36.76 \mathrm{nM}$ versus $23.35 \mathrm{nM}$, respectively) (Supplementary Table S1). Overall, among the five drugs tested, the parasites were most susceptible to ART at a GMIC $_{50}$ of $4.6 \mathrm{nM}$ (Geometric mean $\mathrm{GMIC}_{50}$ [95\% CI] $=4.56$ [3.95., 5.27]); the other four drugs recorded much higher $\mathrm{GMIC}_{50}$ values: CQ (15.5nM), DHA (18.9nM), MFQ (27.3nM) and AMD (42.4nM) in increasing order (Figure 3). Moreover, the $\mathrm{GMIC}_{50}$ ranges for all five test drugs were very wide with the maximum values ranging between 184.3 and $373.3 \mathrm{nM}$.

The pairwise comparison of ex vivo $\mathrm{GMIC}_{50}$ values for the five drugs is shown in Table 2. The correlation between each pair of drugs was significant and positive. The weakest positive correlation was found between MFQ and DHA $(r=0.34 ; \mathrm{p}<0.001)$, followed by MFQ-AMD $(r=0.35 ; \mathrm{p}<0.001)$, DHA-AMD $(r=0.35 ; \mathrm{p}<0.001)$, AMD-CQ $(r=0.39 ; \mathrm{p}<0.001)$, ART-AMD $(r=0.44$; $\mathrm{p}<0.001)$, DHA-CQ $(r=0.49 ; \mathrm{p}<0.001)$, ART-CQ $(r=$ $0.52 ; \mathrm{p}<0.001)$, MFQ-CQ $(r=0.56 ; \mathrm{p}<0.001)$, MFQ-ART $(r=0.58 ; \mathrm{p}<0.001)$ and the strongest between ART and DHA $(r=0.66 ; \mathrm{p}<0.001)$.

\section{Discussion}

A DAPI-based ex vivo assay was carried out to monitor drug sensitivity of $P f$ field isolates' population circulating in two ecological zones in Ghana with different transmission intensities and over a period of 3 years. The success rate recorded in this study was based on the successful ex vivo cultivation of the clinical isolates and all isolates which did not show appreciable growth in culture were eliminated from subsequent analysis as unsuccessful. Interestingly, by eliminating those clinical isolates, the power reduced from 
Table I Ex vivo Susceptibility of Plasmodium falciparum Isolates Against Selected Antimalarial Drugs

\begin{tabular}{|c|c|c|c|c|c|c|}
\hline \multirow[t]{2}{*}{ Drug } & \multicolumn{2}{|c|}{ Assay Success \% (n/N) } & \multirow[t]{2}{*}{ Overall Assay Success \% (n/N) } & \multirow[t]{2}{*}{ Geometric Mean IC $\mathrm{C}_{50}(\mathrm{nM})[95 \% \mathrm{Cl}]$} & \multicolumn{2}{|c|}{ GMIC $_{50}$ Range (nM) } \\
\hline & Begoro & Cape Coast & & & Minimum & Maximum \\
\hline Chloroquine & $58(70 / 120)$ & $65(136 / 210)$ & $62(206 / 330)$ & $15.45[13.70,17.44]$ & 2.1 & 353.4 \\
\hline Amodiaquine & $47(56 / 120)$ & $53(1 \mid 8 / 210)$ & $53(174 / 330)$ & $42.42[36.22,49.67]$ & 0.9 & 184.3 \\
\hline Dihydroartemisinin & $47(56 / / 20)$ & $56(135 / 210)$ & $58(191 / 330)$ & $18.93[16.91,21.20]$ & 0.3 & 235.6 \\
\hline Artesunate & $63(75 / 120)$ & $51(107 / 210)$ & $55(182 / 330)$ & $4.56[3.95 ., 5.27]$ & 0.5 & 283.7 \\
\hline Mefloquine & $61(73 / 120)$ & $67(140 / 210)$ & $65(213 / 330)$ & $27.28[22.93,32.44]$ & 1.8 & 373.3 \\
\hline
\end{tabular}

$90 \%$ to $80 \%$ but that was still enough to differentiate the levels between the two sites. Among the five drugs tested, the parasites were most susceptible to ART whereas much higher mean $\mathrm{GMIC}_{50}$ values were observed for the other four drugs with amodiaquine recording the highest $\mathrm{GMIC}_{50}$. Moreover, the $\mathrm{GMIC}_{50}$ ranges for all five test drugs were very wide with high maximum values and this could probably be explained by the challenges associated with ex vivo studies of which multiple clones of parasites may be present and their sensitivities to the test drugs may differ. ${ }^{31}$ Further studies might be required to ascertain the sources of these high $\mathrm{GMIC}_{50}$ ranges. The $\mathrm{GMIC}_{50}$ values for CQ, ART, and DHA were significantly higher for Cape Coast isolates compared to Begoro. Positive correlations were found in the pairwise comparison of test drugs and they were all statistically significant. A measure of positive correlation in the responses between two individual antimalarial drugs may suggest a cross-resistance, but in these instances, the correlation must be strong. The positive correlation observed in this study, although significant, they were all very weak except that between ART and DHA which was not surprising because these two drugs belong to the class of antimalarials ${ }^{31}$ sharing the same metabolite for their action. DHA-MFQ correlation was the weakest and too low to suggest a possible cross-resistance between them.

In Ghana, ART-AMD is one of the first-line drugs for the treatment of uncomplicated malaria and is also used to treat pregnant women after the first trimester. ${ }^{32}$ Efficacy surveillance of the individual drugs used for the combination therapies is important for detecting the possible development of drug resistance among the $P f$ parasites circulating in the country and preventing its dissemination. The study found Pf parasites as most susceptible to ART at a mean $\mathrm{GMIC}_{50}$ that is comparable to those reported by other drug-efficacy studies $^{22,29,33}$ in West Africa. However, the wide range of $\mathrm{GMIC}_{50}$ values estimated for ART raises a cause for concern. Moreover, the partner drug AMD recorded an $\mathrm{GMIC}_{50}$ much higher than what was estimated in some other studies. ${ }^{29,34,35}$ A similar ex vivo study conducted in Senegal also recorded higher $\mathrm{GMIC}_{50}$ values for AMD. ${ }^{36}$ Also, a significantly positive correlation was found between ART and AMD which may be indicative of cross-resistance between the drugs. However, to protect the prolonged efficacy of ART, it is essential for the partner drug in the combination therapy to be clinically effective. ${ }^{29}$ For instance, artesunate-mefloquine treatment failure in Cambodia was correlated with in vitro mefloquine resistance. ${ }^{14}$ Hence, continual monitoring of the efficacy of AMD is crucial as parasite resistance to AMD has already been reported in the African region. ${ }^{12,13}$

The $P f$ parasites showed a higher tolerance for DHA in the current study compared to drug responses reported earlier in Ghana, ${ }^{22}$ Senegal $^{29,33,34,36,37}$ and among patients hospitalized in France with imported malaria from malaria-endemic countries including Ghana. ${ }^{35}$ Although the reduced susceptibility of the parasite to DHA may not be indicative of resistance, it is important that this drug is closely monitored as it is a partner drug in one of the alternative artemisinin combination therapies in Ghana for uncomplicated malaria (dihydroartemisinin piperaquine). Besides, the emerging resistance to artemisinin in South-East Asia ${ }^{8,9,17}$ coupled with the artemisininresistant strains identified in some studies in Senegal ${ }^{37-39}$ corroborates the need for further exploring parasite sensitivity to artemisinin in the country. DHA was positively correlated with both MFQ and ART although the correlation with the former was weaker. Zatra et $\mathrm{al}^{40}$ also found a high prevalence of parasite isolates with reduced susceptibility to DHA but a strong correlation between DHA and MFQ. The correlation found between ART-DHA in this study was the highest with variation in parasite susceptibility to DHA accounting for over $60 \%$ of variation in parasite response to ART. The high coefficient of determination suggests cross-resistance between these two drugs hence the need for a reinforced surveillance of antimalarial drug resistance in Ghana. 

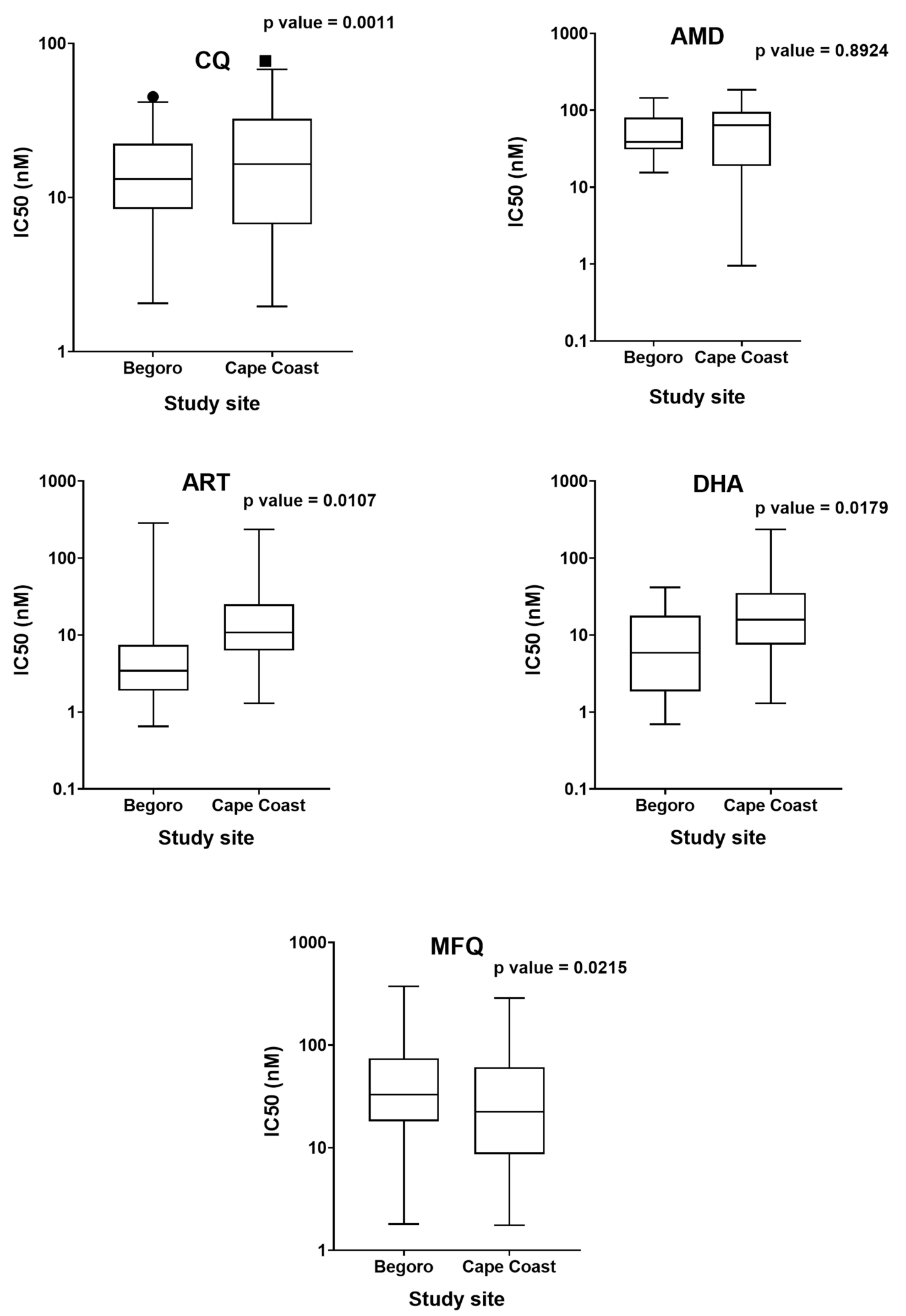

Figure 2 Distribution of GM IC 50 values of chloroquine (CQ), amodiaquine (AMD), dihydroartemisinin (DHA), artesunate (ART) and mefloquine (MFQ) against P. falciparum parasites collected from Cape Coast and Begoro. The dot and square in CQ panel indicate single IC $\mathrm{C}_{50}$ value outliers for Begoro and Cape Coast, respectively. 
CQ

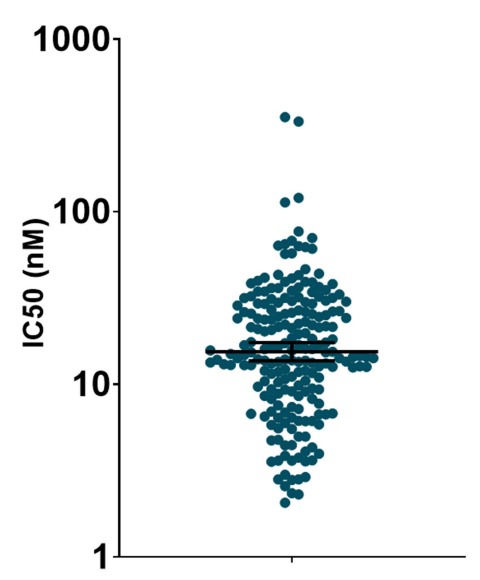

ART

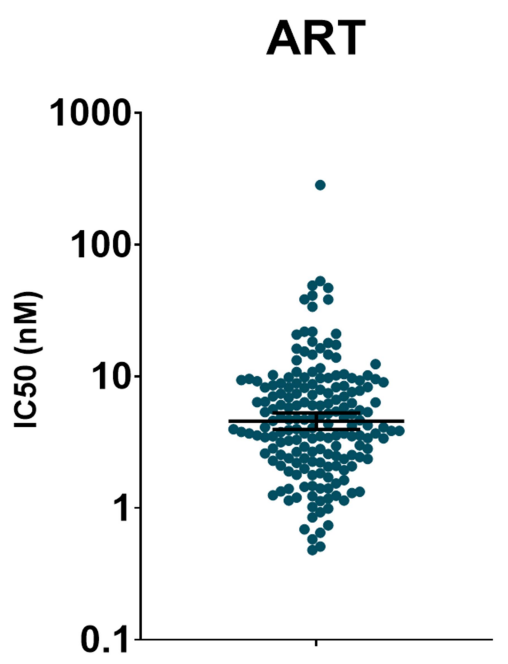

AMD

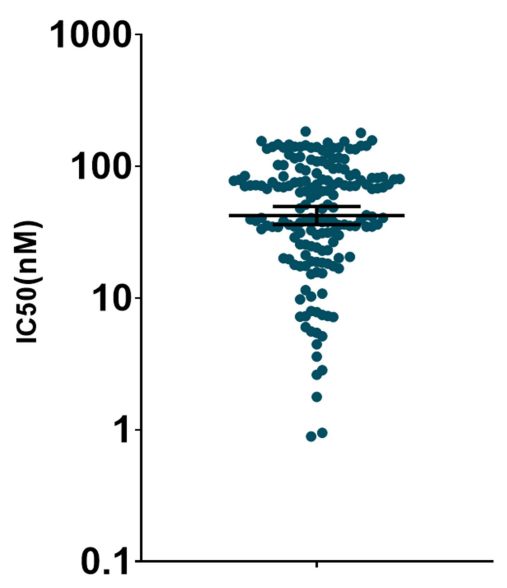

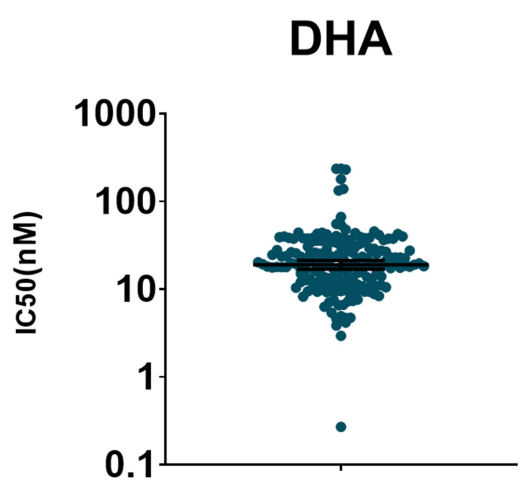

\section{MFQ}

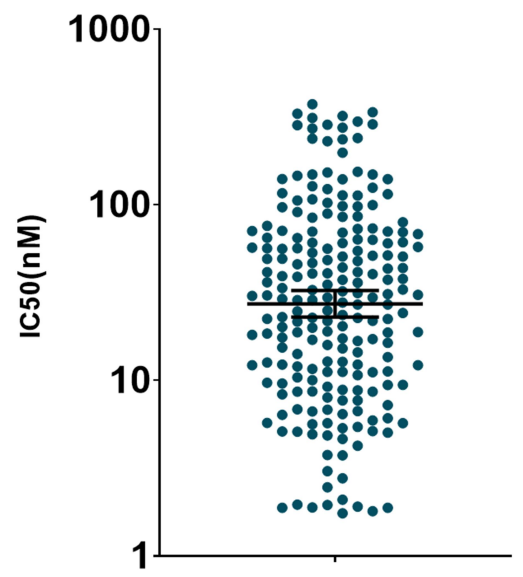

Figure 3 Distribution of $\mathrm{GMIC}_{50}$ values of Plasmodium falciparum in vitro susceptibility against selected antimalarial drugs. The green dots represent individual IC ${ }_{50}$ value of each parasite isolate and the horizontal lines represent the geometric mean of $\mathrm{IC}_{50}$ and the $95 \%$ confidence intervals $(\mathrm{CI})$. 
Table 2 Pairwise Correlation of ex vivo $\mathrm{GMIC}_{50}$ Values for Test Drugs

\begin{tabular}{|l|l|l|l|l|l|}
\hline & Chloroquine & Amodiaquine & Dihydroartemisinin & Artesunate & Mefloquine \\
\hline Chloroquine & $\mathrm{I}$ & & & & \\
Amodiaquine & $0.393^{* * *}$ & $\mathrm{I}$ & & \\
Dihydroartemisinin & $0.4850^{* * *}$ & $0.3548^{* * *}$ & $\mathrm{I}$ & \\
Artesunate & $0.5232^{* * *}$ & $0.4410^{* * *}$ & $0.657 \mathrm{I}^{* * *}$ & $\mathrm{I}$ & \\
Mefloquine & $0.555 \mathrm{I}^{* * *}$ & $0.3544^{* * *}$ & $0.338 \mathrm{I}^{* * *}$ & $0.5803^{* * *}$ & $\mathrm{I}$ \\
\hline
\end{tabular}

Note: ${ }^{* * *}$ p value $<0.001$

Mefloquine is not a recommended drug for the treatment of malaria in Ghana but it serves as one of the major prophylactic drugs used by non-immune travelers to the country, hence its inclusion in the assessment. The $\mathrm{GMIC}_{50}$ estimated for MFQ is comparable to some previous estimations ${ }^{34,37}$ and higher than others. $^{22,35}$ A positive correlation was found between MFQ and AMD drug responses although weak $(r=0.35)$, MFQ and CQ $(r=0.56)$ as well as MFQ and ART $(r=0.58)$. The clinical and epidemiological inferences for these correlations observed cannot be hastily drawn as MFQ is not used much in Ghana. Further monitoring of the evolution of $P f$ susceptibility to MFQ, and correlations of clinical outcomes with pharmacokinetic and phenotypic responses as well as with molecular markers $^{34}$ need to be established to identify any trends in MFQ resistance considering that prophylaxis failure with MFQ has been previously reported in Senegal. ${ }^{41}$

A large improvement in the efficacy of chloroquine was observed in the present study which is consistent with other reports from previous assessments $22,29,33-36$ in West Africa. This is corroborated by the significant decline in the prevalence of $P f \mathrm{CQ}$ resistance markers in Ghana. ${ }^{42,43}$ This improved sensitivity of $P f$ isolates to CQ observed parallels the withdrawal of CQ and introduction of ACTs countrywide in 2005. This is not peculiar to Ghana as the resurgence in CQ sensitive parasites has been observed in West Africa. ${ }^{29,44}$ Notwithstanding, resistance to significant number of antimalarial drugs has been associated with genetic polymorphisms observed in $P f$ single nucleotide polymorphisms (SNPs) in pfcrt and pfmdr1, which affect responses to multiple drugs. A single mutation, pfert 76T, is a known mediator of resistance to $\mathrm{CQ}$ and $\mathrm{AMD} .^{45}$ In the African region two common mutations of pfmdr1, $86 \mathrm{Y}$ and $1246 \mathrm{Y}$ have been linked to a decreased sensitivity to both CQ and AMD. ${ }^{46}$ A recent study conducted in the same study sites in Ghana did show a steady decline in the prevalence of chloroquine resistance mediator pfcrt $76 \mathrm{~T}$ with about $95 \%$ prevalence rate of pfcrt K76 chloroquine-sensitive strains. ${ }^{43}$
The $\mathrm{GMIC}_{50}$ values for CQ, ART, and DHA were significantly higher for Cape Coast samples compared to Begoro. Although malaria transmission at Cape Coast is expected to be low to moderate compared to Begoro, the transmission pattern could be complicated by the differences in both environmental and socioeconomic conditions which could impact the development of drug sensitivity. Cape Coast receives most of its clients from surrounding communities with poor infrastructural development coupled with the presence of stagnant water which could contribute significantly to mosquito breeding and an increase in malaria transmission. The resultant increased anti-malarial use could introduce a surge in drug pressure in these areas. ${ }^{22}$ Also, factors such as intrinsic human genetic factors and illegal drug use among others could influence the re-emergence of sensitive Pf strains. ${ }^{47}$ Besides, there have been reports of the indiscriminate use of anti-malarial drugs in this study area. ${ }^{4,49}$

Reduced sensitivity to drugs in vitro does not necessarily correlate with clinical treatment failure but the patterns in drug response generally reflect parasite capacity to withstand chemotherapy among the population studied. ${ }^{50,51}$ We observed reduced Pf isolates' sensitivities to three (AMD, DHA and MFQ) out of the five drugs used in our assessment compared to previous estimates of $6.11 \mathrm{nM}, 4.0 \mathrm{nM}$ and 10.12 nM for AMD, DHA and MFQ, respectively. ${ }^{22}$ Ghana still has a very high burden of malaria and has recorded an increase in cases. $^{52}$ Although the current efficacy rate of ART-AMD is very high, ${ }^{24}$ about 2 out of 3 Ghanaians self-treat malaria and about a third of suspected malaria cases are treated empirically without parasitological testing. ${ }^{52}$ This calls for more intensified efforts by NMCP in campaigning against improper use of the ACTs and monitoring for drug sensitivity at the sentinel sites that have been set up across the country.

\section{Conclusion}

In conclusion, we have used the ex vivo DAPI assay to assess the sensitivities of clinical parasites isolates collected over 3 consecutive years from two sites of different 
transmission intensities in Ghana to five drugs (single individual drugs). Although we did see higher tolerance levels to most of the drugs used over the period (including the ACTs), this does not mean high drug treatment failures because these were tested for individual drugs most of which are used in combinations in Ghana except chloroquine that is currently not in use in Ghana. This study has clearly demonstrated the continual return of chloroquinesensitive parasites after its withdrawal as first-line drug for the treatment of uncomplicated malaria over a decade ago. It is therefore prudent to use this method to continually monitor these individual drugs in Ghana.

\section{Acknowledgments}

Our sincere gratitude goes to the staff of the Immunology and Epidemiology Department, NMIMR for technical, field and laboratory support. Again, we do express our appreciation to the management and staff of the Begoro Government hospital and Ewim Polyclinic for their support. The Wirth Group at the Harvard T.N. Chan School of Public Health is very much appreciated for donating the drugs as gift to Anita Ghansah. We are also grateful to all the study participants and especially their parents/Guardians at both sites. The study received funding from the NIH (Grant No. 5R01AI099527-02) awarded to Dr Anita Ghansah.

\section{Disclosure}

Emma E. Kploanyi reports grants from NIH, during the conduct of the study. The authors report no other potential conflicts of interest in this work.

\section{References}

1. World Health Organization.World Malaria Report 2020: 20 Years of Global Progress and Challenges. World Health Organisation; 2020. https://www.who.int/publications/i/item/9789240015791.

2. Müller O. Malaria in Africa: Challenges for Control and Elimination in the 21st Century. Peter Lang Frankfurt; 2011.

3. Hyde JE. Drug-resistant malaria. Trends Parasitol. 2005;21 (11):494-498. doi:10.1016/j.pt.2005.08.020

4. Sibley $\mathrm{CH}$. Understanding drug resistance in malaria parasites: basic science for public health. Mol Biochem Parasitol. 2014;195 (2):107-114. doi:10.1016/j.molbiopara.2014.06.001

5. Dondorp AM, Nosten F, Yi P, et al. Artemisinin resistance in Plasmodium falciparum Malaria. $N$ Engl $J$ Med. 2009;361 (5):455-467. doi:10.1056/NEJMoa0808859

6. WHO. Global Report on Antimalarial Drug Efficacy and Drug Resistance: 2000-2010. 2010.

7. Goldberg DE, Siliciano RF, Jacobs WR. Outwitting evolution: fighting drug-resistant TB, Malaria, and HIV. Cell. 2012;148(6):1271-1283. doi:10.1016/j.cell.2012.02.021

8. Noedl H, Se Y, Schaecher K, Smith BL, Socheat D, Fukuda MM. Evidence of artemisinin-resistant malaria in Western Cambodia. $N$ Engl J Med. 2008;359(24):2619-2620. doi:10.1056/nejmc0805011
9. Phyo AP, Nkhoma S, Stepniewska K, et al. Emergence of artemisinin-resistant malaria on the western border of Thailand: a longitudinal study. Lancet. 2012;379(9830):1960-1966. doi:10.1016/ S0140-6736(12)60484-X

10. Na-Bangchang K, Ruengweerayut R, Mahamad P, Ruengweerayut K, Chaijaroenkul W. Declining in efficacy of a three-day combination regimen of mefloquine-artesunate in a multi-drug resistance area along the Thai-Myanmar border. Malar J. 2010;9(1):1-10. doi:10.1186/1475-2875-9-273

11. Beshir K, Sutherland CJ, Merinopoulos I, et al. Amodiaquine resistance in Plasmodium falciparum malaria in Afghanistan is associated with the pfert SVMNT allele at codons 72 to 76. Antimicrob Agents Chemother. 2010;54(9):3714-3716. doi:10.1128/AAC.00358-10

12. Sa JM, Twu O. Protecting the malaria drug arsenal: halting the rise and spread of amodiaquine resistance by monitoring the PfCRT SVMNT type. Malar J. 2010;9(1):374. doi:10.1186/1475-2875-9-374

13. Folarin OA, Bustamante $\mathrm{C}$, Gbotosho GO, et al. In vitro amodiaquine resistance and its association with mutations in pfert and pfmdr1 genes of Plasmodium falciparum isolates from Nigeria. Acta Trop. 2011;120(3):224-230. doi:10.1016/j.actatropica.2011.08.013

14. Rogers WO, Sem R, Tero T, et al. Failure of artesunate-mefloquine combination therapy for uncomplicated Plasmodium falciparum malaria in southern Cambodia. Malar J. 2009;8(1):1-9. doi:10.1186/1475-2875-8-10

15. Wongsrichanalai C, Meshnick SR. Declining artesunate-mefloquine efficacy against falciparum malaria on the Cambodia-Thailand border. Emerg Infect Dis. 2008;14(5):716-719. doi:10.3201/eid1405.071601

16. Witkowski B, Iriart X, Soh PN, et al. pfmdr1 amplification associated with clinical resistance to mefloquine in West Africa: implications for efficacy of artemisinin combination therapies. J Clin Microbiol. 2010;48(10):3797-3799. doi:10.1128/JCM.01057-10

17. Ashley EA, Dhorda M, Fairhurst RM, et al. Spread of artemisinin resistance in Plasmodium falciparum Malaria. $N$ Engl $J$ Med. 2014;371(5):411-423. doi:10.1056/nejmoa1314981

18. Mita T, Tanabe K, Kita K. Spread and evolution of Plasmodium falciparum drug resistance. Parasitol Int. 2009;58(3):201-209. doi:10.1016/j.parint.2009.04.004

19. Petersen I, Eastman R, Lanzer M. Drug-resistant malaria: molecular mechanisms and implications for public health. FEBS Lett. 2011;585 (11):1551-1562. doi:10.1016/j.febslet.2011.04.042

20. Koram K, Quaye L, Abuaku B. Efficacy of amodiaquine/artesunate combination therapy for uncomplicated malaria in children under five years in ghana. Ghana Med J. 2008;42(2):55-60.

21. Adjei GO, Kurtzhals JAL, Rodrigues OP, et al. Amodiaquine-artesunate vs artemether-lumefantrine for uncomplicated malaria in Ghanaian children: a randomized efficacy and safety trial with one year follow-up. Malar J. 2008;7:127-137. doi:10.1186/1475-2875-7-127

22. Quashie NB, Duah NO, Abuaku B, et al. A SYBR Green 1-based in vitro test of susceptibility of Ghanaian Plasmodium falciparum clinical isolates to a panel of anti-malarial drugs. Malar J. 2013;12 (1):1-12. doi:10.1186/1475-2875-12-450

23. Färnert A, Williams TN, Mwangi TW, et al. Transmission-dependent tolerance to multiclonal Plasmodium falciparum Infection. $J$ Infect Dis. 2009;200(7):1166-1175. doi:10.1086/605652

24. Abuaku BK, Mensah BA, Ofori MF, et al. Efficacy of artesunate/ amodiaquine in the treatment of uncomplicated malaria among children in Ghana. Am J Trop Med Hyg. 2017;97(3):690-695. doi:10.4269/ajtmh.15-0826

25. Ghana Statistical Service. 2010 Population and Housing Census: Summary Report of Final Results.; 2012. Available from: https://www. statsghana.gov.gh/gssmain/storage/img/marqueeupdater/Census2010_ Summary_report_of_final_results.pdf. Accessed January 16, 2020.

26. Ministry of Food and Agriculture. Fanteakwa District. Published 2012. Available from: http://mofa.gov.gh/site/sports/districtdirectorates/eastern-region/225-fanteakwa. Accessed February 12 2020. 
27. Abuaku B, Duah N, Quaye L, Quashie N, Koram K. Therapeutic efficacy of artemether-lumefantrine combination in the treatment of uncomplicated malaria among children under five years of age in three ecological zones in Ghana. Malar J. 2012;11(1):388. doi:10.1186/1475-2875-11-388

28. Kweku M, Liu D, Adjuik M, et al. Seasonal intermittent preventive treatment for the prevention of anaemia and malaria in Ghanaian children: a randomized, placebo controlled trial. PLoS One. 2008;3 (12):12. doi:10.1371/journal.pone.0004000

29. Ndiaye D, Patel V, Demas A, et al. Short report: A non-radioactive DAPI-based high-throughput in vitro assay to assess Plasmodium falciparum responsiveness to antimalarials - Increased sensitivity of P. falciparum to chloroquine in senegal. Am J Trop Med Hyg. 2010;82 (2):228-230. doi:10.4269/ajtmh.2010.09-0470

30. Le Nagard H, Vincent C, Mentré F, And JLB. Online analysis of in vitro resistance to antimalarial drugs through nonlinear regression. Comput Methods Programs Biomed. 2011;104(1):10-18. doi:10.1016/j. cmpb.2010.08.003

31. Cui L, Mharakurwa S, Ndiaye D, Rathod PK, Rosenthal PJ. Antimalarial drug resistance: literature review and activities and findings of the ICEMR network. Am J Trop Med Hyg. 2015;93:57-68. doi:10.4269/ajtmh.15-0007

32. Ministry of Health. Anti-Malaria Drug Treatment Policy for Ghana: 2nd Revised Version. 2009. Available from: https://www.ghanahealth service. org/ghs-item-details.php? cid=2\&scid=55\&id=54. Accessed January 16, 2020.

33. Tinto H, Bonkian LN, Nana LA, et al. Ex vivo anti-malarial drugs sensitivity profile of Plasmodium falciparum field isolates from Burkina Faso five years after the national policy change. Malar J. 2014;13(1):1-7. doi:10.1186/1475-2875-13-207

34. Fall B, Diawara S, Sow K, et al. Ex vivo susceptibility of Plasmodium falciparum isolates from Dakar, Senegal, to seven standard anti-malarial drugs. Malar J. 2011;10(1):1-8. doi:10.1186/1475-2875-10-310

35. Pascual A, Parola P, Benoit-Vical F, et al. Ex vivo activity of the ACT new components pyronaridine and piperaquine in comparison with conventional ACT drugs against isolates of Plasmodium falciparum. Malar J. 2012;11(1):45. doi:10.1186/1475-2875-11-45

36. Mbaye A, Gaye A, Dieye B, et al. Ex vivo susceptibility and genotyping of Plasmodium falciparum isolates from Pikine, Senegal. Malar J. 2017;16(1):1-7. doi:10.1186/s12936-017-1897-6

37. Van Tyne D, Dieye B, Valim C, et al. Changes in drug sensitivity and anti-malarial drug resistance mutations over time among Plasmodium falciparum parasites in Senegal. Malar J. 2013;12:1. doi:10.1186/ 1475-2875-12-441

38. Fall B, Pascual A, Sarr FD, et al. Plasmodium falciparum susceptibility to anti-malarial drugs in Dakar, Senegal, in 2010: an ex vivo and drug resistance molecular markers study. Malar J. 2013;12:1. doi:10.1186/1475-2875-12-107

39. Wurtz N, Fall B, Pascual A, et al. Prevalence of molecular markers of Plasmodium falciparum drug resistance in Dakar, Senegal. Malar J. 2012:11. doi:10.1186/1475-2875-11-197.

40. Zatra R, Lekana-douki JB, Lekoulou F, Bisvigou U, Ngoungou EB, Ndouo FST. In vitroantimalarial susceptibility and molecular markers of drug resistance in Franceville, Gabon. BMC Infect Dis. 2012;12 (1):307. doi:10.1186/1471-2334-12-307

Infection and Drug Resistance

\section{Publish your work in this journal}

Infection and Drug Resistance is an international, peer-reviewed openaccess journal that focuses on the optimal treatment of infection (bacterial, fungal and viral) and the development and institution of preventive strategies to minimize the development and spread of resistance. The journal is specifically concerned with the epidemiology of
41. Gari-Toussaint M, Pradines B, Médicale VM-LP. 2002 U. Sénégal et paludisme: echec prophylactique vrai de la méfloquine. Presse Med. 2002;31:1136.

42. Duah NO, Wilson MD, Ghansah A, et al. Mutations in Plasmodium falciparum chloroquine resistance transporter and multidrug resistance genes, and treatment outcomes in Ghanaian children with uncomplicated malaria. $J$ Trop Pediatr. 2007;53(1):27-31. doi: $10.1093 /$ tropej/fml076

43. Mensah BA, Aydemir O, Myers-Hansen JL, et al. Antimalarial drug resistance profiling of plasmodium falciparum infections in ghana using molecular inversion probes and next-generation sequencing. Antimicrob Agents Chemother. 2020;64(4):e01423-19. doi:10.1128/ AAC.01423-19

44. Lu F, Zhang M, Culleton RL, et al. Return of chloroquine sensitivity to Africa? Surveillance of African Plasmodium falciparum chloroquine resistance through malaria imported to China. Parasit Vectors. 2017;10(1):1-9. doi:10.1186/s13071-017-2298-y

45. Fidock D, Nomura T, Talley A. Cell RC-M, 2000 Undefined. Mutations in the P. Falciparum Digestive Vacuole Transmembrane Protein PfCRT and Evidence for Their Role in Chloroquine Resistance. Elsevier; 2021. Available from: https://www.sciencedir ect.com/science/article/pii/S1097276505000778. Accessed January 4.

46. Valderramos S, sciences DF-T in pharmacological, 2006 undefined. Transporters involved in resistance to antimalarial drugs. Elsevier. Available from: https://www.sciencedirect.com/science/article/pii/ S0165614706002240?casa_token=15Iq2MBNqxkAAAAA:eVkCCmAbjafh69cY_SSMJ 89 xe 20_ozWU0WFezUZ_2j 1 AOi9UUMSw9UAJbvLyX_ct8DbLsPORw.. Accessed January 4 2021.

47. Oladipo OO, Wellington OA, Sutherland CJ. Persistence of chloroquine-resistant haplotypes of Plasmodium falciparum in children with uncomplicated Malaria in Lagos, Nigeria, four years after change of chloroquine as first-line antimalarial medicine. Diagn Pathol. 2015;10(1):41. doi:10.1186/s13000-015-0276-2

48. Afoakwah R, Boampong JN, Egyir-Yawson A, Nwaefuna EK, Verner ON, Asare KK. High prevalence of PfCRT K76T mutation in Plasmodium falciparum isolates in Ghana. Acta Trop. 2014;136:32-36. doi:10.1016/j.actatropica.2014.03.030

49. Asare KK. Evaluation of Plasmodium falciparum chloroquine resistant markers in selected health facilities in Central Region after seven years of banning chloroquine treatment in Ghana. 2014. https://erl. ucc.edu.gh/jspui/bitstream/123456789/2768/1/ASARE2014.pdf.

50. Wellems T, Plowe C. Chloroquine-resistant malaria. J Infect Dis. 2001;184:770-776. doi:10.1086/322858

51. Ekland E, Fidock D. In vitro evaluations of antimalarial drugs and their relevance to clinical outcomes. Int $J$ Parasitol. 2008;38:743-747. doi:10.1016/j.ijpara.2008.03.004

52. WHO. World Malaria Report 2019; 2019. https://www.who.int/newsroom/fact-sheets/detail/malaria.

antibiotic resistance and the mechanisms of resistance development and diffusion in both hospitals and the community. The manuscript management system is completely online and includes a very quick and fair peerreview system, which is all easy to use. Visit http://www.dovepress.com/ testimonials.php to read real quotes from published authors.

\section{Dovepress}

